\title{
Safety and efficacy of azacitidine in the treatment of elderly patients with myelodysplastic syndrome
}

This article was published in the following Dove Press journal:

Clinical Interventions in Aging

18 June 2012

Number of times this article has been viewed

\section{Ellen K Ritchie \\ Leukemia Program, Weill Medical College of Cornell University and The New York Presbyterian Hospital, New York, NY, USA}

Correspondence: Ellen K Ritchie Weill Medical College of Cornell University, The New York Presbyterian Hospital, 520 East 68th Street, New York, NY 10065, USA

Tel +l 2127462700

Fax + I 6469621605

Email ritchie@med.cornell.edu
Abstract: The goals of treating older patients with myelodysplastic syndrome (MDS) are different than for younger patients. Few elderly patients are able to pursue an allogeneic stem cell transplant for potential cure of the disease. The focus for the treatment of older patients with MDS is therefore not curative, but rather alleviation of symptoms, improvement in quality of life, maintenance or improvement of functional status, and continued independent living. Prolongation of survival is only important if functional status and quality of life can be maintained, and there is greater risk of losing these outcomes in elderly patients. Azacitidine is an important drug for the treatment of older patients with MDS. Data from the AZA-001 trial has shown a survival benefit for patients with high-risk disease treated with azacitidine. Importantly, treatment has also been shown to improve quality of life for MDS patients. Subset analysis of the data has shown that the drug can be used safely in even the oldest patients with MDS and is superior to treatment with other established regimens, such as low-dose cytarabine. Given the delay between the initiation of treatment and the clinical response, patients may need aggressive supportive care with antiemetics, prophylactic antibiotics, and transfusions to maintain them through therapy. Azacitidine provides a better quality of response when it is used beyond the first response, so ongoing treatment is generally recommended in responding patients. A new oral preparation of the drug is in development that will make the treatment more feasible and comfortable for elderly patients.

Keywords: geriatrics, myelodysplastic syndrome, acute myeloid leukemia, azacitidine, supportive care

\section{Introduction}

Advancing age is the major risk factor for development of myelodysplastic syndrome (MDS). Although MDS can occur at any age, including childhood, the disease primarily affects the elderly with the median onset in the seventh decade of life. SEER data from 2001 to 2003 indicates that $86 \%$ of MDS cases are diagnosed in individuals age 60 years or older. ${ }^{1}$ The estimated incidence of MDS increases significantly with age, ranging from 0.7 per 100,000 adults during the fourth decade of life to 10.8-36.3 per 100,000 adults after age 70 years. There is a five-fold increase in risk between age 60 and 80 years of age. ${ }^{1,2}$ As the population of elderly persons grows (it is estimated that by the year $2025,1.2$ billion people will be aged $\geq 60$ years), the incidence of MDS within the total population will increase. ${ }^{3}$

Elderly patients diagnosed with MDS experience symptoms that can impair quality of life and compromise their ability to accomplish daily activities independently. In older patients, these symptoms can be exacerbated by comorbid 
illness and polypharmacy. The symptoms of the disease are primarily related to cytopenias and can be overwhelming. Depending on the severity and number of cytopenias, patients complain of excessive fatigue, bruising, bleeding, night sweats, bone pain, fever, skin rash, undesired weight loss, and recurrent infections. There have only been a few studies focusing on the symptoms of MDS and their impact on quality of life. ${ }^{4-6}$ These studies identify excessive fatigue as one of the most debilitating symptoms of this disease. The correlation of fatigue with the magnitude of cytopenias has not been consistent. Jansen et al could correlate hemoglobin level with the degree of fatigue and found that improvement in hemoglobin improved symptoms. However, Steensma et al could not find a correlation between the degree of fatigue and the hemoglobin level and surmised that the fatigue experienced by patients may be independent of cytopenias. ${ }^{4}$ Nonetheless, the premise of all treatment modalities is to improve symptoms by raising blood counts. Hellstrom-Lindberg et al investigated the effect of treatment with erythropoiesis-stimulating agents and granulocyte colony-stimulating factor on the quality of life of 36 patients with MDS before and after receiving treatment. ${ }^{7}$ Global quality of life was significantly improved in patients with increased hemoglobin levels in response to treatment $(P=0.01) .^{7}$ Although the aim of available treatments is to improve cytopenia and thereby to improve symptoms, there is no therapy targeted specifically to treating fatigue.

Treatment options for elderly patients with MDS are limited, and there are many factors that increase the likelihood that they may not receive any active MDS treatment. Agerelated comorbid conditions, functional impairment, poor tolerability, ineffectiveness of available therapies, and patient/ family preferences can all influence whether the physician recommends active treatment to a patient. ${ }^{8}$ Results of a large cross-sectional survey of physicians treating older patients with MDS (most patients were over 70 years of age) indicated that $27 \%$ of newly diagnosed patients with higher-risk disease and $24 \%-49 \%$ of patients with established higher-risk disease received supportive care only. ${ }^{9}$ For the older patient, there has been little evidence that active treatment improves functional status and quality of life. ${ }^{10}$ These outcomes have not been the primary focus of treatment studies but are of great importance to elderly patients who are deciding among treatment options for this disease.

\section{MDS biology and aging}

MDS is a myeloid neoplasm that is characterized by abnormal differentiation, morphology, and maturation of hematopoietic cells in the bone marrow. Patients with this disease have greater risk of evolving to acute myeloid leukemia. ${ }^{11}$ Although the disease is characterized by peripheral cytopenias, the bone marrow is usually hypercellular. In recent years, multiple chromosomal and molecular aberrations have been discovered that contribute to the development of this disease. The importance of chromosomal abnormalities in the prognosis of this disease is well established by the incorporation of cytogenetic risk into the International Prognostic Scoring System (IPSS) for MDS. In this system, patients are stratified into four risk groups by the percentage of blasts in the bone marrow, the number and degree of cytopenias at presentation, and by the type of cytogenetic abnormality found in the initial bone marrow sample. ${ }^{12}$ Patients with complex karyotypes (more than three chromosomal abnormalities) and chromosome 7 abnormalities are classified as high-risk and those with isolated deletion $5 \mathrm{q}$ (del5q), isolated deletion 20q (del20q), and loss of the Y chromosome have a more favorable prognosis. However, over $50 \%$ of patients with MDS have a normal karyotype, ${ }^{11}$ which is considered by the International Prognostic Scoring System to have an intermediate outcome. In actuality, the outcome is highly variable in this subset of patients with MDS. Newer techniques in genomic analysis have uncovered molecular changes beyond chromosomal abnormalities that contribute to the pathology of this disease. ${ }^{11}$ For example, Jiang et al examined the mechanism of neoplastic evolution in patients with MDS and acute myeloid leukemia, and found that aberrant DNA methylation was seen in every patient sample. Chromosomal aberrations were seen in $79 \%$ of samples from patients with early/low-grade MDS. Aberrant methylation can cooperate with chromosomal deletions to silence tumor suppressor genes. However, given the ubiquity and extent of aberrant methylation in these samples from patients with MDS and acute myeloid leukemia, it appears that epigenetic aberrations were the dominant mechanism for tumor suppressor gene silencing and clonal variation. ${ }^{13}$ A series of recent papers suggests that splice gene mutations are among the most frequent molecular aberrations in MDS and may define distinct clinical phenotypes. ${ }^{14-17}$ For example, SF3B1-mutated patients present with lower hemoglobin levels, increased white blood cell and platelet counts and are more likely to have DMT3A mutations. SRSF2-mutated patients cluster in RAEB-1 and RAEB-2 subtypes and exhibit pronounced thrombocytopenia. ${ }^{18}$ Mutations in the TP53 tumor suppressor gene can occur in conjunction with these lesions and can lead to inferior outcome. ${ }^{19}$ TET-2, EZH2, and DNMT3a are genes associated with epigenetic regulation, and mutations 
in these genes can lead to better response to hypomethylating agents, such as azacitidine and decitabine. ${ }^{20,21}$ Recent studies of genes that contribute to the development of MDS are important and may lead to the discovery of new agents that target the specific genes causing this disease.

The fact that MDS is a disease of older persons suggests that there may be a correlation between the development of MDS and the process of aging. Pathways that include the processing of messenger RNAs are potential biomarkers of aging. Age-associated disruption in the balance of alternatively expressed isoforms for select genes may be a prominent feature of human aging. ${ }^{22}$ Given the emerging evidence of the connection of mutations involved in RNA processing to the development of some forms of MDS, it is tempting to speculate that there is a connection between the aging process and the development of this disease. Another recent paper by Forsberg et al looked at monozygotic twin cohorts and single-born cohorts to examine their genome changes over time. ${ }^{23}$ They found that in a few healthy subjects, cytogenetic abnormalities characteristic of MDS developed as they aged and included del5q, del20q, and trisomy 8 . None of the subjects with these abnormalities were diagnosed with MDS at the time of the detection. Over time, some of these patients had an increase in the degree of abnormality in their peripheral blood mononuclear cells, but some subjects had a decrease or disappearance. These results suggested to the authors a process of "autocorrection" of the immune system, given that aberrant clones could also disappear from the circulation over time. ${ }^{23}$ Changes in the immune system of patients as they age may contribute to abnormalities that lead to disease. Much work needs to be done to explore further the connections between aging and disease, but new insight into the processes may lead to new therapies directed at the older patient.

\section{Rationale for azacitidine treatment}

The only potentially curative treatment for MDS is allogeneic stem cell transplantation. In a subset of select older patients who have minimal comorbid illness and excellent performance status, allogeneic transplantation can be attempted for a possibility of prolonged overall survival. Given that the majority of older patients are not candidates for transplantation, treatment modalities that improve symptoms, maintain functional abilities and quality of life, as well as extend overall survival are needed. Because aberrant methylation has been established as a ubiquitous lesion in MDS patients, hypomethylating agents have emerged at the forefront. Two hypomethylating agents have been approved for the treatment of MDS, ie, azacitidine and decitabine. The AZA-001 trial was a Phase III, international, multicenter, controlled, open-label trial of patients with higher-risk MDS who were randomly assigned to treatment with azacitidine at $75 \mathrm{mg} / \mathrm{m}^{2} /$ day $\times 7$ days every 28 days or conventional care (including best supportive care, low-dose cytarabine, or intensive chemotherapy). This trial demonstrated both an overall survival benefit and an improvement in quality of life, and established azacitidine as an important treatment for patients with high-risk MDS. ${ }^{24}$ In contrast, decitabine has not been shown to have the same benefit in the treatment of patients with high-grade MDS. Two studies comparing decitabine with best supportive care in high-grade MDS patients using the dose schedule approved by the US Food and Drug Administration $\left(15 \mathrm{mg} / \mathrm{m}^{2}\right.$ intravenously every 8 hours for 3 days) failed to demonstrate a survival benefit. ${ }^{25,26}$ The dosing schedule approved by the Food and Drug Administration administers higher decitabine doses in a more toxic schedule than the more commonly prescribed 5-day schedule developed at the MD Anderson Cancer Center. ${ }^{27}$ The 5-day schedule has not been studied in a randomized trial. ${ }^{28}$ Treatment with either azacitidine or decitabine leads to transfusion independence in $50 \%$ of cases. ${ }^{24-26,28,29}$ Only azacitidine has been shown to confer a survival benefit in randomized clinical trials.

Because a major goal of MDS treatment is to improve quality of life, the measurement of "improvement" is important to treatment choice. The AZA-001 trial incorporated a quality of life measurement. In this trial, quality of life was assessed by telephone interviews conducted at baseline and at days 50,106, and 182. The patients also answered two standard surveys of quality of life, including the European Organization for Research and Treatment of Cancer Quality of Life Questionnaire C30 and the Mental Health Inventory. These measurements were comprehensive and the data showed that patients treated with azacitidine demonstrated improved quality of life as compared with patients in the conventional care arm, with improvement in fatigue, dyspnea, physical functioning, positive affect, and psychological distress. ${ }^{30}$ Given the demonstrable improvements in quality of life coupled with superior overall survival, azacitidine was firmly established as a standard of care in high-risk MDS.

\section{AZA-00I trial Feasibility and tolerability in the oldest patients}

The median age of treated patients on the AZA-001 trial was 69 years. Substudy analysis was done to measure the efficacy and safety in the oldest patients in this 
study (aged 75 years or older). ${ }^{8}$ AZA-001 accrued 87 patients aged years 75 or older. Investigators preselected treatment options for their patients in this trial and the majority chose best supportive care (60 patients) as the option of choice. Investigators chose a more aggressive treatment option for a smaller group of patients, ie, low-dose cytarabine in 24 patients and intensive chemotherapy in three patients. After randomization, 38 patients were treated with azacitidine, 33 patients with best supportive care, 14 patients with low-dose cytarabine and two patients with intensive chemotherapy. Azacitidine improved overall survival in this "older elderly" cohort (hazards ratio $0.48, P=0.019$ ). A similar percentage of older patients became transfusion-dependent because the overall study population (43\%) and hospitalizations for adverse events were not more frequent in the azacitidine cohort. Grade 3 and 4 anemia, neutropenia, and thrombocytopenia with azacitidine versus the conventional treatment cohort were $13 \%$ versus $4 \%, 61 \%$ versus $17 \%$, and $50 \%$ versus $30 \%$, respectively. Azacitidine was efficacious and tolerable in these older patients. ${ }^{8}$ Given the improvement in overall survival, transfusion dependence, and tolerability, the drug emerged as an excellent treatment choice, even in the oldest patients presenting with high-grade MDS.

However, older patients with MDS are not often offered treatment for their disease because of comorbid illness or poor performance status. The AZA-001 trial did not enroll patients with a poor performance status (Eastern Cooperative Oncology Group [ECOG] stage 3 or 4) or who had therapy-related MDS. The contribution of age and performance status to azacitidine response was evaluated in a paper by Itzykason et al. ${ }^{31}$ In that study, 282 patients with high-risk MDS were enrolled on a compassionate program for azacitidine treatment before the drug was commercially available. The median age of these patients was 71 years, and 56 patients had a performance status of ECOG 2 or worse. The authors developed a prognostic scoring system to predict which patients would respond to azacitidine treatment by retrospectively studying this cohort. Interestingly, age and therapy-related disease were not factors in whether patients would respond to azacitidine. The authors found previous low-dose cytarabine $(P=0.009)$, bone marrow blasts $>15 \%$ $(P=0.004)$, and abnormal karyotype $(P=0.03)$ predicted lower response rates. A complex karyotype predicted a shorter duration of response $(P=0.0003)$. Decreased overall survival was predicted by performance status ECOG 2 or worse, intermediate-risk and poor-risk cytogenetics, presence of circulating blasts, and red blood cell transfusion greater than 4 units in 8 weeks. ${ }^{31}$ The prognostic score generated from this paper was applied to patients enrolled on the AZA-001 for validation of results. In this study, performance status was an important factor determining response to azacitidine. However, analysis of performance status in older MDS patients can be misleading. Some patients have decreases in performance status because of comorbid illness and the development of MDS occurs in the context of other debilitating illnesses. In these patients, it is not clear that improving cytopenias will substantially improve performance status. Other older patients become debilitated as a result of the cytopenias caused by MDS. Whether these patients have improvements in performance status with treatment is not well documented, and is an important factor in deciding whether patients with poor performance status may benefit from treatment. Missing from studies of older patients are the effects of comorbid illness on response to azacitidine. Future prospective studies need to look more carefully at the relationships between performance status, comorbid illness, and cytopenias to help determine whether treatment of older patients with poor performance status can improve the ability to accomplish activities of daily living and improve quality of life.

In addition, it would also be helpful going forward to determine the number of hospitalized patients who are discharged to subacute rehabilitation facilities versus to home. Although these are not common outcomes in treatment trials for cancer patients, these outcomes are very relevant to older patients. Determination of these outcomes should be addressed in future prospective trials.

\section{Direct comparison with low-dose cytarabine in elderly patients}

The AZA-001 trial was neither designed nor powered to compare survival between azacitidine and any other conventional care arms, including low-dose cytarabine. Low-dose cytarabine had been a standard of care for treatment of higher-grade MDS prior to the introduction of azacitidine in elderly patients. ${ }^{10}$ There had been speculation since the publication of results from AZA-001 that the impact of azacitidine may not be different from that of low-dose cytarabine in patients with MDS. A recent analysis compared patients who were preselected by their physicians for treatment with low-dose cytarabine but who were randomized to receive azacitidine on the trial. Forty-five patients were randomized to azacitidine and 49 patients were randomized to low-dose cytarabine. Patient characteristics were well balanced between the two arms. In keeping with the overall trial population, the azacitidine-treated patients 
(who had been preselected by their physicians for low-dose cytarabine) had twice the 2-year survival as the low-dose cytarabine-treated patients. The difference in outcome was most pronounced for patients with poor-risk cytogenetics, in particular those with abnormalities of chromosome 7 . Hematologic improvements were more common and durable in the azacitidine-treated cohort, and these subjects had fewer days of hospitalization. ${ }^{32}$

\section{Patients with acute myeloid leukemia on AZA-00I}

Older patients who present with acute myeloid leukemia may not be good candidates for intensive chemotherapy for their disease. Intensive chemotherapy in this population is associated with high mortality rates and poor overall survival at two years. ${ }^{33}$ In patients with acute myeloid leukemia, less intensive alternatives to standard chemotherapy have been an active area of investigation. The AZA-001 trial included 113 patients with blast counts in the bone marrow between $20 \%$ and $30 \%$ who would be classified as having acute myeloid leukemia in accordance with the World Health Organization nomenclature. These patients were preassigned by their physicians to best supportive care $(n=63)$, low-dose cytarabine $(n=34)$, or standard induction chemotherapy $(n=16)$. Of these 113 patients, 55 were assigned to azacitidine and 58 to the preselected treatment option. The baseline characteristics were similar between the two groups. Interestingly, the difference in 2-year overall survival for azacitidine-treated patients was greater for this group than in the overall cohort (50\% versus $16 \%$, median survival 24.5 versus 16 months). For patients with acute myeloid leukemia and low bone marrow blast counts, azacitidine appears to be an effective treatment, with superior median survival and lower 30-day mortality than standard induction therapy in older patients. ${ }^{33}$ Kantarjian et al, in a paper looking at the results of intensive chemotherapy induction in 998 patients over 65 years with acute myeloid leukemia and MDS, found that the overall complete remission rate was $45 \%$ with an induction mortality of $29 \%$. One-year survival of this group of patients with acute myeloid leukemia depended on whether they were categorized as favorable, intermediate, or unfavorable risk. Patients in the favorable risk group had a one-year survival of $50 \%$ but those in the unfavorable group had $10 \%$ survival. The azacitidine-treated patients as a group had superior median survival and a lower induction mortality of $11 \%$ in the first three months of treatment than older patients treated with intensive induction in the MD Anderson series. ${ }^{33}$ The data suggest a substantial benefit to some patients with acute myeloid leukemia treated with azacitidine and will need to be tested in prospective trials. ${ }^{32}$ The efficacy of this therapy in patients with more proliferative acute myeloid leukemia remains the subject of ongoing investigation.

\section{Treatment schedule for azacitidine}

A major difficulty of azacitidine treatment in older patients is that the drug must be given subcutaneously or intravenously for several consecutive days on a monthly basis. For many patients, this means numerous visits to the physician's office to receive treatment. In addition, they must rely on a caregiver to assist them in transportation to and from the physician's office, particularly when they have symptomatic disease or are suffering adverse effects of chemotherapy. Efforts have been made to make the schedule of treatment less difficult for patients, caregivers, and physicians. The treatment schedule on the AZA-001 trial was azacitidine $75 \mathrm{mg} / \mathrm{m}^{2}$ per day for 7 days every 28 days. ${ }^{24}$ The treatment was given subcutaneously, but the Food and Drug Administration has also approved intravenous administration based on the fact the drug has been shown to have the same bioavailability. ${ }^{34}$ The schedule used in AZA-001 was found to be feasible; $86 \%$ of patients had no dose reduction, and $80 \%$ of cycles were given at 4-5-week intervals without prophylactic use of myeloid growth factor. Patients treated on this schedule did not have higher rates of infection or bleeding relative to the observation/conventional therapy groups. ${ }^{24}$

There have been alternative dosing schedules proposed with similar response rates to the schedule used in AZA001, but none of these schedules has been shown to improve overall survival. ${ }^{35}$ An alternative schedule of $50 \mathrm{mg} / \mathrm{m}^{2}$ for 5 days every 4 weeks has been used in patients with lowerrisk disease with the goal of improved quality of life/reduced transfusions, and is reasonable if circumstances make it difficult to treat with the 7 -day schedule. ${ }^{35}$ In a retrospective study of azacitidine use in community practices, the 7-day schedule may be associated with a better response, so the validity of the 5-day schedule is controversial. ${ }^{36}$ Although the 7-day schedule is inconvenient for clinician and patients, given the survival benefit and tolerability data from AZA-001, higher-risk patients should be treated on the 7-day schedule whenever possible.

Difficulties with the treatment schedule could be improved with an oral preparation of the drug. This would make the treatment more accessible for older patients because it is easier and would not require trips to the physician's office for administration. The negative effects of injection and of intravenous placement would also be alleviated. An oral prepara- 
tion of azacitidine is in development and has been studied in a Phase I dose-escalation study using the 7-day schedule. At the maximum tolerated dose, oral bioavailability of parenteral azacitidine was $13 \%$. Surprisingly, methylation reversal was similar to that seen with parenteral azacitidine, although fewer loci were significantly demethylated. Seventy-three percent of previously untreated patients developed clinical responses. ${ }^{37}$ This oral preparation is currently being studied in other dosing schedules to test the concept that prolonged administration of lower doses of hypomethylating drugs may provide better responses. Approval of an oral drug with the same efficacy as the current preparations would presumably make treatment of older MDS patients more feasible and comfortable with fewer complications.

\section{Supportive care of patients treated with azacitidine}

There can be a prolonged period of time between the initiation of azacitidine treatment and the clinical response. Before blood counts improve, patients may have a long period of pancytopenia. Clinicians must understand the side effects of treatment and be able to support patients through a period of prolonged cytopenias. Important data on supportive treatment of patients receiving azacitidine treatment were obtained from two studies, ie, CALGB 9221 and AZA-001..$^{24,38,39}$ These data are summarized in Table 1. In both of these studies, most of the adverse events took place in the first two cycles of treatment before improvement in blood counts and tended to decrease in frequency during subsequent cycles. In subgroups of patients in both trials who completed 6 or more treatment cycles, a similar pattern of reduced frequencies of adverse events with each successive treatment was seen. Cytopenias were ubiquitous in both trials. In AZA-001 and CALGB 9221, more patients experience neutropenia, anemia, or thrombocytopenia during the first

Table I Symptomatic management of patients treated on azacitidine

\begin{tabular}{ll}
\hline Symptom & Management \\
\hline Injection site reaction & $\begin{array}{l}\text { Warm compression, antihistamines, steroids, } \\
\text { topical steroids } \\
\text { Laxatives }\end{array}$ \\
Constipation & $\begin{array}{l}\text { Antiemetics } \\
\text { Trausea }\end{array}$ \\
Anemia & $\begin{array}{l}\text { erythropoiesis-stimulating agents on clinical trial } \\
\text { Transfusion or romiplostim on clinical trial }\end{array}$ \\
Thrombocytopenia & $\begin{array}{l}\text { Granulocyte colony-stimulating factor short } \\
\text { course, prophylactic antibiotics, and antifungals }\end{array}$ \\
Feutropenia & Intravenous antibiotics to cover broad spectrum
\end{tabular}

or two treatment cycles. Nadir for hematologic parameters occurred at a median of 15-16 days in CALGB 9221 and 14-15 days in AZA-001. Overall, in AZA-001 and CALGB $9221,78 \%$ and $89 \%$ of patients, respectively, had anemia, thrombocytopenia, or neutropenia. Ten patients in AZA-001 and 19 patients on CALGB 9221 experienced fever with median durations of 5-7 days. Worsening of symptoms of fatigue was seen in patients in both trials, with median durations of 8 days on AZA-001 and 33 days on CALGB 9221. In AZA-001, the infection rates per patient year were not statistically different in the azacitidine group versus the conventional care group. Events associated with azacitidine administration included injection site reactions in $29 \%$ of AZA-001 patients and in 3.3\% of patients on CALGB 9221. These involved erythema and discomfort, and were transient. Patients also reported gastrointestinal events. Constipation was the most frequently reported gastrointestinal event on AZA-001 (50.3\%), and most occurrences were in the first two cycles of treatment and may have been exacerbated by antiemetic regimens. The median duration of constipation was 8 days in AZA-001 and 17 days in CALGB 9221. Nausea was the most frequently reported gastrointestinal event on CALGB 9221. Grade 3 and 4 gastrointestinal events were reported by less than $6 \%$ of patients in either study.

Strategies used by both studies to manage anemia, thrombocytopenia, and neutropenia associated with azacitidine treatment included delay of the next treatment cycle, dose reduction, and blood product transfusions. In AZA-001, $86 \%$ of patients had no dose adjustments, with a median cycle length of 28 days. Twenty-nine percent of cytopenias were managed by drug dose delay and $9 \%$ by dose reduction. Anemia was managed with transfusion in $87 \%$ of cases and thrombocytopenia was managed by transfusion in $29 \%$ of cases. In $15 \%$ of cases, febrile neutropenia was managed with antibiotics. In cases of severe neutropenia and fever, a brief course of granulocyte colony-stimulating factor was added. In CALGB 9221, cytopenias resulted in dose interruptions and reductions in $23 \%$ and $11 \%$ of patients, respectively. The median duration of cycle length was 34.3 days.

In AZA-001, nausea, vomiting, constipation, and diarrhea were managed with antiemetics, laxatives, stool softeners, and antidiarrheals. Less than $12 \%$ of injection reactions were treated with corticosteroids or antihistamines. Little benefit was observed with the use of topical medications. In AZA-001, 99\% of patients received concomitant medications. The most common were paracetamol, furosemide, ondansetron, allopurinol, and levofloxacin. The most common intravenous antibiotics used were 
vancomycin, piperacillin/tazobactam, and gentamicin. Thirteen percent of patients were treated with granulocyte colony-stimulating factor.

In CALGB 9221, the most common concomitant medications were antiemetics. Other medications used frequently included analgesics, systemic antibacterials, and systemic antihistamines. No patients were treated with granulocyte colony-stimulating factor because the medication was prohibited on the study.

Growth factor therapy with erythropoietin-stimulating agents and granulocyte-stimulating agents are used as primary treatment in low-grade MDS to improve cytopenias. To enhance efficacy and decrease the burden of transfusion during azacitidine treatment, there have been studies looking at the efficacy of combination therapy. Growth factors have been used in combination with azacitidine with some promising results. Itzykson et al retrospectively studied a cohort of 282 patients with high-risk MDS who received azacitidine in a compassionate program, including 32 patients who received concomitant erythropoiesis-stimulating agents. Forty-four percent of patients treated with azacitidine who received erythropoiesis-stimulating agents and $29 \%$ who did not receive this therapy achieved hematologic improvement in their anemia $(P=0.07)$. Transfusion independence was achieved in $48 \%$ versus $20 \%$ and median overall survival was longer in the group treated with an erythropoiesis-stimulating agent (19.6 months versus 11.9 months, $P=0.04) .{ }^{40}$ The results need to be confirmed in a prospective study of this combination. Azacitidine has also been used in conjunction with the thrombopoietin mimetic, romiplostim, in a Phase II study. ${ }^{41}$ Forty-five patients with low-risk and intermediaterisk MDS were stratified by baseline platelet counts and randomized to romiplostim at two dose levels versus placebo subcutaneously during the first four cycles of azacitidine treatment. The endpoint was incidence of clinically significant thrombocytopenic events. The numbers were too small to find significance in the clinical endpoints, but the data suggested that romiplostim may provide clinical benefit during azacitidine therapy. Patients in this study seemed to increase median platelet counts over time (3-4 months) and seemed to have a reduced need for platelet transfusion during azacitidine treatment. The combination of growth factors with azacitidine to reduce transfusion during treatment and improve outcome needs further investigation.

\section{Azacitidine in low-risk MDS}

For older patients with low-risk MDS, treatment options include growth factor therapy with erythropoiesis-stimulating agents and granulocyte colony-stimulating factor, ${ }^{7}$ and treatment with the immunomodulating agent, lenolidomide. ${ }^{42,43}$ Azacitidine has also been studied in patients with low-risk MDS. ${ }^{44}$ Musto et al studied a retrospective cohort of patients who had low-risk disease and were treated through a compassionate-use named patient program in Italy. Seventyfour patients with a median age of 70 years were studied. Among these patients, 84\% were transfusion-dependent, $57 \%$ received erythropoiesis-stimulating agents, and 51\% were older than 70 years. Patients received a median of seven cycles on three different treatment schedules. The overall response rate was $45.9 \%$ (complete remission $10.8 \%$, partial response $9.5 \%$, hematologic improvement $20.3 \%$ ). In patients who completed four cycles, the overall response rate was higher at $51.6 \%$. The median duration of response was 6 (range 1-30) months. There was a survival benefit seen in responders versus nonresponders (94\% versus 54\% of patients projected to be alive at 2.5 years, $P<0.0014) .{ }^{44}$ The results of this retrospective study suggest that azacitidine may be an effective treatment for low-grade MDS that can confer a survival benefit in responders. The results need confirmation in a prospective study.

\section{Conclusion}

The goals of care in the elderly patients are different than for younger patients with this disease. Younger patients are treated with a curative goal, and there is a willingness to accept more toxic therapies including allogeneic stem cell transplantation. For older patients, particularly those with comorbid illness and compromised performance status, aggressive strategies are often not possible. For these patients, the goal of treatment is to improve symptoms, maintain or improve functional status, and prolong independent survival. For many older patients, prolongation of overall survival is meaningful only if quality of life and independence are maintained. Studies of agents to treat MDS have not emphasized the outcomes of functional status and quality of life. Azacitidine has become a standard of care for treatment of patients with MDS, in part because the AZA-001 trial showed benefit in overall survival, together with quality of life for even the most elderly subgroup in the trial. Most adverse events occur in the first two cycles of therapy. Patients need supportive care during this time to treat side effects of treatment and the adverse effects of prolonged cytopenia. The addition of growth factor therapy to azacitidine treatment to improve outcome and decrease transfusions during treatment requires further study. Fortunately, patients who achieve a response need less support in subsequent cycles. At the present time, 
patients who have a response should be continued on the drug until they relapse. More study needs to be done as to how long treatment should be continued after a response is attained.

The benefit of azacitidine treatment in elderly MDS patients has expanded the potential use for this drug. Azacitidine is now being studied in older patients with acute myeloid leukemia as an alternative to intensive chemotherapy. It is also being explored as maintenance therapy for patients treated with intensive chemotherapy for high-grade MDS or acute myeloid leukemia who achieve a complete remission ${ }^{45}$ in combination with growth factors, lenolidomide, and histone deacetylase inhibitors to improve response and increase the duration of response in MDS patients. ${ }^{41,46-49}$

In future trials of agents to treat MDS in elderly patients, especially those who are not candidates for stem cell transplantation, emphasis should be placed not only on achieving prolonged overall survival and a complete remission, but on outcomes of quality of life and functional status. Older patients need to know if treatments will interfere with their ability to accomplish activities of daily living and to live independently. Knowing whether there is an improvement in functional status when cytopenias improve would allow many older patients to make better choices about their MDS treatment. The economic value of using expensive drugs to treat older patients with this disease would be enhanced by concrete data showing improvements in quality of life and function in the older population. These endpoints cannot be neglected in future investigations of these agents and other novel agents to treat this disease.

\section{Disclosure}

The author reports no conflict of interest in this work.

\section{References}

1. United States Cancer Statistics: 1999-2007 incidence and mortality webbased report. Atlanta, GA: Centers for Disease Control and Prevention. Available from: http://apps.nccd.cdc.gov/uscs/. Accessed April 16, 2012.

2. Ma X, Does M, Raza A, Mayne ST. Myelodysplastic syndromes: incidence and survival in the United States. Cancer. 2007;109(8): 1536-1542.

3. US Census Bureau Current Population Reports. 1193.

4. Steensma DP, Heptinstall KV, Johnson VM, et al. Common troublesome symptoms and their impact on quality of life in patients with myelodysplastic syndromes (MDS): results of a large Internet-based survey. Leuk Res. 2008;32(5):691-698.

5. Jansen AJ, Essink-Bot ML, Beckers EA, Hop WC, Schipperus MR, Van Rhenen DJ. Quality of life measurement in patients with transfusiondependent myelodysplastic syndromes. Br J Haematol. 2003;121(2): 270-274.
6. Platzbecker U, Hofbauer LC, Ehninger G, Holig K. The clinical, quality of life, and economic consequences of chronic anemia and transfusion support in patients with myelodysplastic syndromes. Leuk Res. January 31 , 2012. [Epub ahead of print.]

7. Hellstrom-Lindberg E, Gulbrandsen N, Lindberg G, et al. A validated decision model for treating the anaemia of myelodysplastic syndromes with erythropoietin + granulocyte colony-stimulating factor: significant effects on quality of life. Br J Haematol. 2003;120(6): 1037-1046.

8. Seymour JF, Fenaux P, Silverman LR, et al. Effects of azacitidine compared with conventional care regimens in elderly ( $\geq 75$ years) patients with higher-risk myelodysplastic syndromes. Crit Rev Oncol Hematol. 2010;76(3):218-227.

9. Sekeres MA, Schoonen WM, Kantarjian H, et al. Characteristics of US patients with myelodysplastic syndromes: results of six cross-sectional physician surveys. J Natl Cancer Inst. 2008;100(21):1542-1551.

10. Burnett AK, Milligan D, Prentice AG, et al. A comparison of low-dose cytarabine and hydroxyurea with or without all-trans retinoic acid for acute myeloid leukemia and high-risk myelodysplastic syndrome in patients not considered fit for intensive treatment. Cancer. 2007;109(6): 1114-1124.

11. Shih AH, Levine RL. Molecular biology of myelodysplastic syndromes. Semin Oncol. 2011;38(5):613-620.

12. Greenberg P, Cox C, LeBeau MM, et al. International scoring system for evaluating prognosis in myelodysplastic syndromes. Blood. 1997;89(6): 2079-2088.

13. Jiang Y, Dunbar A, Gondek LP, et al. Aberrant DNA methylation is a dominant mechanism in MDS progression to AML. Blood. 2009;113(6): 1315-1325.

14. Ward AJ, Cooper TA. The pathobiology of splicing. J Pathol. 2010; 220(2):152-163.

15. Yoshida K, Sanada M, Shiraishi Y, et al. Frequent pathway mutations of splicing machinery in myelodysplasia. Nature. 2011;478(7367): 64-69.

16. Visconte V, Makishima H, Jankowska A, et al. SF3B1, a splicing factor is frequently mutated in refractory anemia with ring sideroblasts. Leukemia. 2012;26(3):542-545.

17. Papaemmanuil E, Cazzola M, Boultwood J, et al. Somatic SF3B1 mutation in myelodysplasia with ring sideroblasts. $N$ Engl J Med. 2011;365(15):1384-1395.

18. Damm F, Kosmider O, Gelsi-Boyer V, et al. Mutations affecting mRNA splicing define distinct clinical phenotypes and correlate with patient outcome in myelodysplastic syndromes. Blood. 2012;119(14):3211-3218.

19. Jadersten M, Saft L, Smith A, et al. TP53 mutations in low-risk myelodysplastic syndromes with $\operatorname{del}(5 \mathrm{q})$ predict disease progression. J Clin Oncol. 2011;29(15):1971-1979.

20. Ko M, Huang Y, Jankowska AM, et al. Impaired hydroxylation of 5-methylcytosine in myeloid cancers with mutant TET2. Nature. 2010;468(7325):839-843.

21. Jankowska AM, Makishima H, Tiu RV, et al. Mutational spectrum analysis of chronic myelomonocytic leukemia includes genes associated with epigenetic regulation: UTX, EZH2, and DNMT3A. Blood. 2011;118(14):3932-3941.

22. Harries LW, Hernandez D, Henley W, et al. Human aging is characterized by focused changes in gene expression and deregulation of alternative splicing. Aging Cell. 2011;10(5):868-878.

23. Forsberg LA, Rasi C, Razzaghian HR, et al. Age-related somatic structural changes in the nuclear genome of human blood cells. Am J Hum Genet. 2012;90(2):217-228.

24. Fenaux P, Mufti GJ, Hellstrom-Lindberg E, et al. Efficacy of azacitidine compared with that of conventional care regimens in the treatment of higher-risk myelodysplastic syndromes: a randomised, open-label, phase III study. Lancet Oncol. 2009;10(3):223-232.

25. Kantarjian H, Issa JP, Rosenfeld CS, et al. Decitabine improves patient outcomes in myelodysplastic syndromes: results of a phase III randomized study. Cancer. 2006;106(8):1794-1803. 
26. Lubbert M, Suciu S, Baila L, et al. Low-dose decitabine versus best supportive care in elderly patients with intermediate- or highrisk myelodysplastic syndrome (MDS) ineligible for intensive chemotherapy: final results of the randomized phase III study of the European Organisation for Research and Treatment of Cancer Leukemia Group and the German MDS Study Group. J Clin Oncol. 2011;29(15): 1987-1996.

27. Steensma DP, Baer MR, Slack JL, et al. Multicenter study of decitabine administered daily for 5 days every 4 weeks to adults with myelodysplastic syndromes: the alternative dosing for outpatient treatment (ADOPT) trial. J Clin Oncol. 2009;27(23):3842-3848.

28. Kantarjian H, Oki Y, Garcia-Manero G, et al. Results of a randomized study of 3 schedules of low-dose decitabine in higher-risk myelodysplastic syndrome and chronic myelomonocytic leukemia. Blood. 2007;109(1):52-57.

29. Silverman LR, McKenzie DR, Peterson BL, et al. Further analysis of trials with azacitidine in patients with myelodysplastic syndrome: studies 8421, 8921, and 9221 by the Cancer and Leukemia Group B. J Clin Oncol. 2006;24(24):3895-3903.

30. Kornblith AB, Herndon JE 2nd, Silverman LR, et al. Impact of azacytidine on the quality of life of patients with myelodysplastic syndrome treated in a randomized phase III trial: a Cancer and Leukemia Group B study. J Clin Oncol. 2002;20(10):2441-2452.

31. Itzykson R, Thepot S, Quesnel B, et al. Prognostic factors for response and overall survival in 282 patients with higher-risk myelodysplastic syndromes treated with azacitidine. Blood. 2011;117(2):403-411.

32. Fenaux P, Gattermann N, Seymour JF, et al. Prolonged survival with improved tolerability in higher-risk myelodysplastic syndromes: azacitidine compared with low dose ara-C. Br J Haematol. 2010;149(2): 244-249.

33. Kantarjian H, O'Brien S, Cortes J, et al. Results of intensive chemotherapy in 998 patients age 65 years or older with acute myeloid leukemia or high-risk myelodysplastic syndrome: predictive prognostic models for outcome. Cancer. 2006;106(5):1090-1098.

34. Marcucci G, Silverman L, Eller M, Lintz L, Beach CL. Bioavailability of azacitidine subcutaneous versus intravenous in patients with the myelodysplastic syndromes. J Clin Pharmacol. 2005;45(5):597-602.

35. Lyons RM, Cosgriff TM, Modi SS, et al. Hematologic response to three alternative dosing schedules of azacitidine in patients with myelodysplastic syndromes. J Clin Oncol. 2009;27(11):1850-1856.

36. Garcia R, de Miguel D, Bailen A, et al. Different clinical results with the use of different dosing schedules of azacitidine in patients with myelodysplastic syndrome managed in community-based practice: effectiveness and safety data from the Spanish azacitidine compassionate use registry (Abstract 2773). Blood. 2009;144(22).
37. Garcia-Manero G, Gore SD, Cogle C, et al. Phase I study of oral azacitidine in myelodysplastic sydromes, chronic myelomonocytic leukemia and acute myeloid leukemia. J Clin Oncol. 2011;29(18):2521-2527.

38. Silverman LR, Demakos EP, Peterson BL, et al. Randomized controlled trial of azacitidine in patients with the myelodysplastic syndrome: a study of the Cancer and Leukemia Group B. J Clin Oncol. 2002;20(10):2429-2440.

39. Santini V, Fenaux P, Mufti GJ, et al. Management and supportive care measures for adverse events in patients with myelodysplastic syndromes treated with azacitidine*. Eur J Haematol. 2010;85(2):130-138.

40. Itzykson R, Thepot S, Beyne-Rauzy O, et al. Does addition of erythropoiesis stimulating agents improve the outcome of higherrisk myelodysplastic syndromes treated with azacitidine? Leuk Res. 2011;36(4):397-400.

41. Kantarjian HM, Giles FJ, Greenberg PL, et al. Phase 2 study of romiplostim in patients with low- or intermediate-risk myelodysplastic syndrome receiving azacitidine therapy. Blood. 2010;116(17):3163-3170.

42. List A, Kurtin S, Roe DJ, et al. Efficacy of lenalidomide in myelodysplastic syndromes. N Engl J Med. 2005;352(6):549-557.

43. List A, Dewald G, Bennett J, et al. Lenalidomide in the myelodysplastic syndrome with chromosome 5q deletion. N Engl J Med. 2006;355(14): 1456-1465.

44. Musto P, Maurillo L, Spagnoli A, et al. Azacitidine for the treatment of lower risk myelodysplastic syndromes: a retrospective study of 74 patients enrolled in an Italian named patient program. Cancer. 2010;116(6):1485-1494.

45. Grovdal M, Karimi M, Khan R, et al. Maintenance treatment with azacytidine for patients with high-risk myelodysplastic syndromes (MDS) or acute myeloid leukaemia following MDS in complete remission after induction chemotherapy. Br J Haematol. 2010;150(3): 293-302.

46. Braiteh F, Soriano AO, Garcia-Manero G, et al. Phase I study of epigenetic modulation with 5-azacytidine and valproic acid in patients with advanced cancers. Clin Cancer Res. 2008;14(19):6296-6301.

47. Sekeres MA, List AF, Cuthbertson D, et al. Phase I combination trial of lenalidomide and azacitidine in patients with higher-risk myelodysplastic syndromes. J Clin Oncol. 2010;28(13): 2253-2258.

48. Sekeres MA, O'Keefe C, List AF, et al. Demonstration of additional benefit in adding lenalidomide to azacitidine in patients with higher-risk myelodysplastic syndromes. Am J Hematol. 2011;86(1):102-103.

49. Pollyea DA, Kohrt HE, Gallegos L, et al. Safety, efficacy and biological predictors of response to sequential azacitidine and lenalidomide for elderly patients with acute myeloid leukemia. Leukemia. October 28, 2011. [Epub ahead of print.]
Clinical Interventions in Aging

\section{Publish your work in this journal}

Clinical Interventions in Aging is an international, peer-reviewed journal focusing on evidence-based reports on the value or lack thereof of treatments intended to prevent or delay the onset of maladaptive correlates of aging in human beings. This journal is indexed on PubMed Central, MedLine, the American Chemical Society's 'Chemical Abstracts Ser-

\section{Dovepress}

vice' (CAS), Scopus and the Elsevier Bibliographic databases. The manuscript management system is completely online and includes a very quick and fair peer-review system, which is all easy to use. Visit http://www.dovepress.com/testimonials.php to read real quotes from published authors. 